\title{
Évaluer la mise en forme du lien écologique
}

\section{Dominique Cottereau}

\section{(2) OpenEdition}

\section{Journals}

Édition électronique

URL : http://journals.openedition.org/ere/6894

DOI : 10.4000 /ere.6894

ISSN : 2561-2271

Éditeur

Centr'ERE

Référence électronique

Dominique Cottereau, «Évaluer la mise en forme du lien écologique», Éducation relative à

l'environnement [En ligne], Volume 2 | 2000, mis en ligne le 15 septembre 2000, consulté le 17 avril

2021. URL : http://journals.openedition.org/ere/6894 ; DOI : https://doi.org/10.4000/ere.6894

Ce document a été généré automatiquement le 17 avril 2021 


\title{
Évaluer la mise en forme du lien écologique
}

\author{
Dominique Cottereau
}

1 Entre le contrôle d'un acquis en termes de savoir et l'appréciation des effets d'un processus éducatif, il y a autant de distance qu'entre l'instruction et l'éducation. Dans le premier l'évaluation se réduit souvent à un jeu de questions - réponses, les unes devant être en parfaite adéquation avec les autres; dans le second l'évaluation est un processus complexe, plein d'incertitude et de risque, car toute situation éducative implique autant d'opérations provoquées que d'interférences qui lui échappent. Dans le champ de l'éducation relative à l'environnement, on est bien dans cette situation délicate de devoir évaluer non seulement une transmission de savoirs, mais aussi des savoir-faire, des attitudes, et même des valeurs. L'éducation à l'environnement agit sur les rapports de l'homme au monde dont les racines s'entremêlent au cœur de son existence affective. Pour transformer des attitudes et habitudes dommageables à l'environnement, il faut plus qu'un enseignement des sciences de la terre et de la vie, plus qu'un discours informatif sur l'actualité de l'environnement, il faut une véritable éducation de l'être global pris dans l'enchevêtrement de ses multiples dépendances naturelles et culturelles. Comment trouver les critères objectifs pour évaluer cette complexité existentielle? Quelles précautions prendre pour ne pas "engrillager" l'humain? Comment ne pas le réduire à quelques attitudes, le sectionner en champs de compétences, lui prêter des intentions qui ne seraient que la projection de ses propres fantasmes? Comment éviter de concentrer un semblant de résultat dans une toute petite partie des informations collectées?

2 Pourtant, bien que la tâche soit mal aisée, on a envie de connaître la portée de son action pédagogique. On voit dans l'évaluation un puissant moyen de réduction de ses erreurs et, surtout, on se dit que l'autre, l'apprenant, a quelque chose à nous dire sur nos méthodes, nos comportements, nos choix d'éducateur. Évaluer c'est aussi écouter, être attentif à la personne insérée dans le système formatif que l'on a établi pour elle.

3 C'est ce travail d'évaluation que j'ai tenté de mettre en œuvre sur mon terrain des classes d'environnement (Cottereau, 1995). Notre structure professionnelle accueille 
des classes de l'enseignement élémentaire, en séjour de deux ou trois semaines, au bord de la mer, pour développer une conscience environnementale chez ces jeunes élèves de 10-12 ans. Nous avions pour postulat que pour modifier le regard des enfants sur l'environnement il ne suffit pas de leur faire comprendre les fonctionnements et dysfonctionnements des écosystèmes littoraux, mais que cet apprentissage cognitif doit s'accompagner d'un éveil de la sensibilité à ses ambiances et à ses paysages. Comment respecter un milieu s'il nous est indifférent ? Comment s'engager dans un changement de comportement plus citoyen vis-à-vis du milieu quand trop d'objectivité et de froideur nous l'ont affectivement éloigné ? Le « mauvais » état de l'environnement dans le monde ne provient-il pas justement de cette trop grande distanciation que l'homme moderne a créée entre lui et la nature (Berque, 1996) ? Une pédagogie du sensible, de l'émotionnel et de l'imaginaire semble devoir être introduite pour permettre une réelle rencontre avec l'environnement et l'établissement d'un lien "écologique " (de la logique de l'habitat). C'est cette mise en forme relationnelle que nous voulions évaluer.

Pour la clarté de l'article je suivrai le processus présenté par Postic et de Ketele (1994) pour qui l'évaluation, c'est :

\section{Énoncer un objectif}

5 Le nôtre est de permettre à l'enfant de créer une relation de type écologique (se sentir chez soi) par la pédagogie de l'alternance écoformatrice. Celle-ci met en parallèle des méthodes rationnelles d'étude du milieu (compréhension de l'écosystème littoral) et des méthodes subjectives d'appropriation affective du même milieu (jeu libre, écriture poétique, art, petit milieu personnel, etc.) (Cottereau, 1999). Le concept d'écoformation traduit tout ce champ relationnel par lequel l'oïkos participe à la formation d'un être, ou d'une personne, tout autant que l'être transforme son oïkos (G. Pineau et coll., 1992).

\section{Énoncer les critères de l'évaluation}

6 L'enfant montrera, dans ses paroles et dans ses jeux, qu'il progresse d'une relation initiale distanciée et objective avec le milieu vers une relation finale d'écodépendance sensible. Je choisis pour observer cela deux modèles qui me permettraient de vérifier ce trajet relationnel des enfants :

7 D'une part, je devrais pouvoir observer une mise en relation de type systémique entre l'enfant et l'environnement. Si la mise en forme écologique s'opérait cela pouvait être sur le modèle de la rencontre de deux systèmes par le biais de leurs interactions. Le modèle organisationnel d'Edgard Morin selon lequel « nul corps, nul objet ne peut être conçu en dehors des interactions qui l'ont constitué, et des interactions auxquelles il participe nécessairement » (1977, p. 56) allait être mon premier outil d'évaluation :

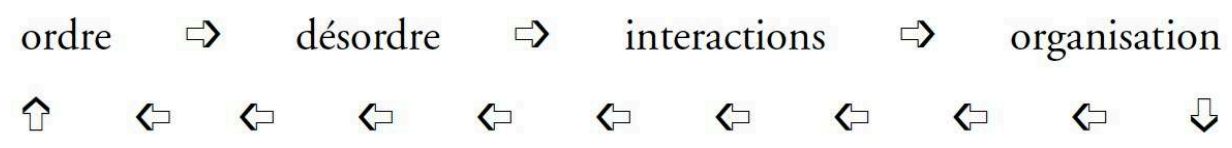

8 Verrons-nous, à l'instar de ce modèle qui doit être perçu en spirale s'élevant au-dessus de son socle (comme un escalier en colimaçon), une progression dans la relation des enfants avec le milieu qui suivrait cette logique d'un ordre initial (l'état préalable du 
rapport enfant - bord de mer), dans lequel surgit un désordre (l'événement classe d'environnement et milieu inhabituel), qui permet de nouvelles interactions (autres perceptions, autres actions, autres rencontres), facilitant alors l'émergence d'une nouvelle organisation relationnelle enrichie et sensible, aboutissant ainsi à un nouvel ordre de l'être dans son rapport au monde ? D'autre part, puisque je supposais une mise en lien affective, ce système écologique instauré par la pédagogie choisie devait pouvoir exprimer son symbolisme. C'est-à-dire que d'une expression neutre et objective sur la mer et son milieu, les enfants devaient passer à un langage plus subjectif, imagé, symbolique, voire poétique. Et pour vérifier cela j'avais besoin de croiser le modèle de Morin avec les outils du symbolisme et de la poétique (Eliade, 1980 ; Bachelard, 1989 ; Durand, 1992). Les enfants transformeront-ils un espace, au préalable neutre, en un écosystème anthropologique plein de souffle, d'objets connus puis reconnus, bruissant, odorant, éclatant de mille correspondances intimes sans fin renouvelées?

\section{Déterminer les informations à recueillir, et les recueillir}

Au cours d'une classe de mer de 21 jours, avec des élèves de 10 à 12 ans, trois techniques évaluatives vont me permettre de recueillir le matériel informatif: l'exercice de rédaction d'un texte libre sur le thème de «la mer » en début et en fin de séjour pour tous les élèves; quatre entretiens interindividuels sous forme semidirective avec deux enfants choisis au hasard, au cours desquels la tâche principale est de parler sur l'emploi du temps écoulé; des observations de ces deux mêmes enfants que j'effectuerai sur les temps de récréation sur la plage, sans guide, en notant tout ce que mon regard pourrait accrocher, et ceci le plus discrètement possible. Pour être réellement dans une situation évaluative de mon action, il aurait fallu que, pour ces deux dernières situations, je m'intéresse aussi à tous les élèves de la classe. Or, la constitution du rapport écologique que je veux évaluer ne peut se percevoir si je regarde toute la classe. Il se révèle dans des instants privilégiés, un mot échappé, un geste esquissé, un mouvement ébauché qui échapperaient à ma vigilance si mon regard et mon écoute se portaient sur l'ensemble du groupe. Ce ne sont pas toujours les moments les plus bruyants qui portent les messages. "Ce n'est pas en pleine lumière, c'est au bord de l'ombre que le rayon, en se diffractant, nous confie ses secrets » nous dit Bachelard (1986, p. 241). Les secrets de l'entre-deux écologique ne se disent bien que dans l'intimité, dans le retrait des regards indiscrets. Nous ne les entendons qu'en prêtant une veille attentive aux instants qui se succèdent sans en laisser échapper une étincelle, cherchant à appréhender aussi bien le non-dit, l'insu, l'ineffable, que le dit. Voilà pourquoi le choix d'une double évaluation: quantitative et sur toute la classe pour ce qui est de la comparaison des textes écrits (Cottereau, 1994), qualitative et avec deux élèves en ce qui concerne les observations et les entretiens.

\section{Confronter informations recueillies et critères posés}

10 En fin de séjour, j'avais devant moi des textes, des enregistrements et des notes... une multitude d'informations qu'il me fallait trier, comparer, analyser. Dans le domaine du non formel, aucune information n'est a priori inutile à la compréhension de l'ensemble. C'est pourquoi je gardai toutes les phrases, tous les mots, tous les gestes que chaque 
enfant exprima. Et je me mis à les classer à la fois le long du modèle organisationnel de Morin et dans le tableau à deux colonnes paroles objectives/paroles subjectives au fur et à mesure du temps passé. Tout ce qui n'y rentrait pas, je décidais de le mettre à part, ce qui s'avéra peu fréquent.

\section{Sortir du désordre}

11 Le modèle de Morin se justifia pour les deux enfants. L'ordre initial de Pierre est un rapport ludique d'immersion avec l'eau. Son texte initial et son premier entretien abondent des plaisirs de la baignade, et ses premiers jeux se déroulent tous dans les premières vaguelettes de l'eau. Océane possède une relation première plus complexe et déjà fort symbolique. Son texte libre est poétique et renvoie, ainsi que ses jeux, à des attirances multiples et profondes (l'eau et la plage, le ciel et les profondeurs etc.). Malgré cela, la nouveauté de la situation classe d'environnement fait surgir le désordre dans ces ordres initiaux. Pierre n'aime ni les paysages, ni les plages du lieu d'accueil. Ses entretiens se focalisent sur des souvenirs anciens plus heureux, il s'échappe, dès qu'il peut, des commentaires sur l'emploi du temps ou exprime le désir de pratiquer des activités qu'il maitrise mieux. Océane choisit de jouer dans la chambre plutôt que sur la plage. Elle parle peu, sauf à manifester des anxiétés face au vent, à la mer, à la pratique de la voile. Puis, avec le temps, Pierre organise la riposte et entre en interaction volontaire avec le milieu. Ses jeux sortent de l'eau, mais tout proche, il élève des murs de pierres qui semblent repousser les vagues. Il ne garde en mémoire, lors des entretiens, que les activités dans lesquelles un brin de courage et d'effort lui ont permis de réussir une tâche. De son côté, Océane sort de sa chambre et se met à explorer les plages de haut en bas, de droite à gauche comme si son corps avait besoin de s'approprier l'espace et le volume du lieu. De temps en temps elle s'arrête et contemple les vagues, furtivement mais très régulièrement. L'entretien devient plus animé et ses paroles se concentrent sur des images de vent, de vagues, d'oiseaux, de voile dont elle commence à apprécier la pratique. La rencontre s'effectue. Pour Pierre l'organisation nouvelle qui en surgit se focalise sur l'estran (zone de balancement des marées). Il se met à collecter des coquillages, des pierres, à fouiller les fissures de rochers. Il construit des châteaux de sable d'une grande organisation architecturale, dans lesquels tout est prévu pour que la vie biologique se développe et que même ses bassins « alimentent la mer ». Le dernier entretien est plein de cette rencontre nouvelle. Le ton de Pierre est gai, léger. Il parle de tout, de ses jeux, de ses plaisirs et des savoirs scientifiques qu'il a construits. Océane, elle aussi, libère sa parole, équilibre les sujets de conversation, entre images et savoirs, entre affectif et objectivité. Sur la plage elle joue avec les vagues, contemple les oiseaux, écoute le vent. L'organisation finale d'Océane est une relation aérienne avec les éléments marins.

\section{La rencontre établie}

Plus loin encore, leurs derniers textes écrits témoignent mieux que l'échange oral ce qu'est devenue cette relation. Pierre, d'un seul trait (il me le dira après) remplit la page dès la consigne formulée, "écrivez ce que vous avez envie d'écrire sur la mer »:

Quand la mer se déchaîne, rien ne peut l'arrêter. Même les chênes si forts et si robustes n'y arrivent pas. Plus rien ne fait le poids. Et quand le vent souffle, c'est pareil. C'est une bataille entre deux titans. 
Mais quand la mer est d'huile, les tuiles des maisons ne volent plus de tous les côtés. Même la guerre cesse. Pollution et bidons, tout est réduit en poussière, une sorte de poussière d'étoiles, d'or, d'argent, de mille rubis diamants. Même le vent devient soleil.

Magnifique recomposition de son parcours. Tout y est: du désordre (Pierre aime beaucoup les arbres), de la riposte engagée (entre deux titans), et de la nouvelle organisation qui en sort, calme et féconde, riche (pleine de ces pierres et coquillages qu'il ramassait). Pierre me dit lors du dernier entretien «je ramasse des pierres blanches, que je vais ramener à Paris pour les mettre sous un microscope. Je suis sûr qu'à l'intérieur il y a des boules qui brillent tout le temps ». Explorer les profondeurs, les intériorités, c'est chercher l'en-soi des choses. «Toute connaissance de l'intimité des choses est immédiatement un poème ", écrit Bachelard (1948, p. 11). Pierre eut cette facilité d'écriture qui donne l'impression que les mots poétiques précèdent la pensée. Océane eut ce même élan poétique. Son premier texte, déjà, offrait une peinture esthétique de la mer : «La mer est belle, bleue, tellement bleue qu'on pourrait la confondre avec le ciel (...) ». Mais en forme de prose et de l'ordre de la relation à l'objet, il s'écrivait à la troisième personne du singulier. Dans le dernier écrit, le poème devient dialogue avec la mer :

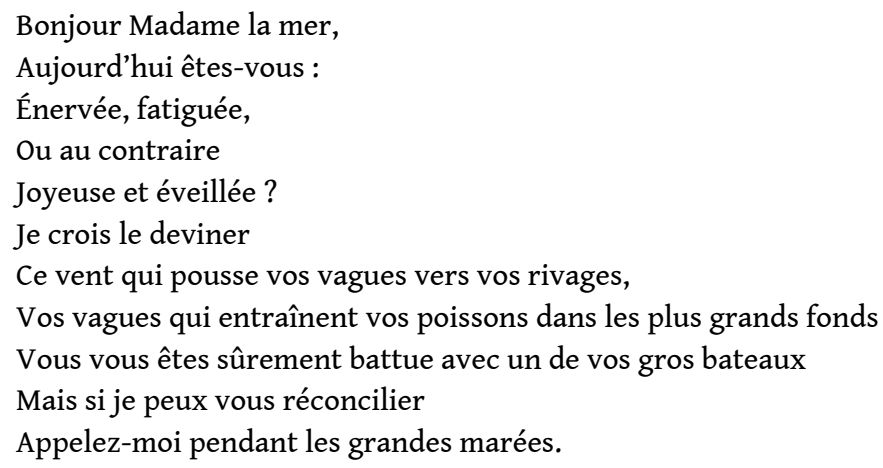

14 La rencontre est là, sincère, entendue, comprise, complice et réciproque. On aurait tort d'y voir un banal animisme ou un jeu de forme. Le tutoiement ou le vouvoiement de la nature témoigne bien plus d'un tête-à-tête engagé, le «je-tu» fonde le monde de la relation (Buber, 1959); une relation pleine d'avenir et peut-être de défense mutuelle. Elle sait qu'elle y reviendra si elle peut réconcilier les contraires... Sans doute est-ce trop tôt pour parler d'écocitoyenneté, mais dans l'histoire écologique d'Océane quelque chose s'est produit de l'ordre du sensible, de l'écoute des éléments, de l'attachement à un milieu. Plus tard, quand l'enfance irriguera sa vie d'adulte, peut-être Océane aura-telle ce comportement respectueux de l'environnement.

\section{Complexité de l'évaluation}

Bien sûr, un tel protocole d'évaluation n'est pas sans obstacle dont particulièrement celui de la subjectivité de l'évaluateur. Mais qui prétend à l'objectivité quand il est impliqué dans le processus pédagogique évalué, celui-là n'est pas humain. La subjectivité est là, et mieux vaut l'éclairer et l'intégrer à l'analyse comme facteur influant plutôt que de la réfuter. Néanmoins cette subjectivité n'est pas celle de «l'Ego métaphysique, fondement et juge suprême de toute chose. C'est le sujet vivant, aléatoire, insuffisant, vacillant, modeste, qui introduit sa propre finitude " (Morin, 1986, p. 22). Elle fait partie de la complexité de l'évaluation. Je sais que mon propre 
rapport sensible à l'environnement maritime a agi secrètement sur le processus. Je sais aussi que le fait d'avoir fait parler ces deux enfants dans des entretiens privilégiés a accéléré leur prise de conscience dans l'établissement d'une relation forte avec le milieu. Je sais encore que mon comportement d'écoute et de grande vigilance favorisa leur mise en confiance dans l'environnement. Mais être conscient de ces facteurs permet de comprendre ce qui est à insérer dans la pédagogie quotidienne: une sensibilité non seulement à l'environnement, mais aussi à l'apprenant. "La sensibilité est une valeur à redécouvrir. Non pas une sorte de sentimentalité ou de mollesse, mais au contraire une fermeté douce qui est portée par une vague de tendresse compréhensive pour l'enfant, l'élève, l'étudiant, le stagiaire adulte " (Barbier, 1997, p. 89). Ne serait-ce pas l'écoute "rogérienne» qu'il nous faudrait retrouver? Cette attitude qui permet d'être réceptif à l'autre, disponible. Écouter est de l'ordre de l'expérientiel plus que de l'expérimental, c'est-à-dire qu'à côté de nos facultés logiques et cognitives, nous mettons en œuvre nos facultés d'intuition et d'imagination. Être à la fois en attitude positive vis-à-vis de l'autre, tout en étant séparé et indépendant de lui : lui permettre d'être ce qu'il est. Sentir le monde de l'autre «comme s'il était le vôtre, mais sans jamais oublier la qualité de "comme si" (Rogers, 1970, p. 204). Nous serions des pédagogues dans le juste milieu de soi et de l'autre, de l'objectif et du subjectif.

Il est bien court notre trajet pédagogique avec ces enfants : trois semaines. Est-ce assez pour que notre évaluation témoigne d'un réel changement? La reliance écologique a besoin de répétitions, de confirmations, de nouveaux couplages. Le "vous" de la rencontre d'Océane peut pâlir, se dissiper, redevenir l'objet neutre, échappé de la relation. Ce n'est que peu à peu que s'éclaircit de proche en proche la conscience du partenariat.

Les rencontres intimes de l'enfant avec le feu, l'air, la terre, l'eau ne s'arrêtent pas à l'enfance. Elles le fécondent de germes, à la fois vitamines de croissance globale, mais aussi embryon à croissance spécifique, avec des temps de gestation propre, pouvant avorter ou aboutir. (Pineau, 1994, p. 11)

Il faudrait revoir Pierre et Océane dix ans plus tard pour achever notre évaluation. Mais si du moins ce court séjour a permis d'engranger quelques images fondatrices, quelques expériences formatrices, des souvenirs odorants, une partie de ceux-là constituera la personnalité de Pierre et celle d'Océane, tous les deux si bien nommés. Le souvenir heureux d'une rencontre est une eau souterraine qui, inépuisablement, irrigue le cours de la vie.

\section{BIBLIOGRAPHIE}

Bachelard, G. (1948). La terre et les rêveries du repos. Paris, France : José Corti.

Bachelard, G. (1986). La formation de l'esprit scientifique. Paris, France : Vrin.

Bachelard, G. (1989). La poétique de la rêverie. Paris, France : Presses Universitaires de France. 
Barbier, R. (1997). L'approche transversale. L'écoute sensible en sciences humaines. Paris, France : Anthropos.

Buber, M. (1959). La vie en dialogue. Paris, France : Aubier-Montaigne.

Berque, A. (1996). Être humains sur la terre. Paris, France : Gallimard.

Cottereau, D. (1994). À l'école des éléments. Écoformation et classe de mer. Lyon, France : Chronique sociale.

Cottereau, D. (1995). Éducation à l'environnement et classe de mer. Instauration du dialogue écologique par une pédagogie de l'écoformation. Thèse de doctorat en Sciences de l'éducation et de la formation. Université François Rabelais, Tours, France.

Cottereau, D. (1999). Chemins de l'imaginaire. Pédagogie de l'imaginaire et éducation à l'environnement. La Caunette, France : Éditions de Babio.

Durand, G. (1992). Les structures anthropologiques de l'imaginaire. Paris, France : Dunod.

Eliade, M. (1980). Images et symboles. Paris, France : Gallimard.

Morin, E. (1977). La méthode. 1. La nature de la nature. Paris, France : Seuil.

Morin, E. (1986). La méthode. 3. La connaissance de la connaissance. Paris, France : Seuil, Points.

Pineau, G. et coll. (1992). De l'air. Essai sur l'écoformation. Paris, France : Païdeia.

Pineau, G. (1994). Pour une éconaissance. Préface In D. Cottereau. À l'école des éléments. Lyon, France : Chronique sociale.

Postic, M. et de Ketele, J.M. (1994). Observer les situations éducatives. Paris, France : Presses Universitaires de France.

Rogers, C.R. (1970). Le développement de la personne. Paris, France : Dunod.

\section{AUTEUR}

\section{DOMINIQUE COTTEREAU}

Dominique Cottereau est diplômée d'un doctorat en Sciences de l'éducation et de la formation. Elle travaille au sein de l'association Échos d'Images pour le développement de l'écoformation dans les domaines de la formation, de l'éducation et de la recherche-action. 